\title{
Precision Medicine and its Role in the Treatment of Sepsis: A Personalised View
}

\author{
Alexandra Lazăr ${ }^{1}$, Anca Meda Georgescu2 ${ }^{*}$, Alexander Vitin ${ }^{3}$, Leonard Azamfirei ${ }^{1}$ \\ 1 Department of Anesthesiology and Intensive Care, University of Medicine, Pharmacy, Science and Technology of \\ Târgu Mureș, Romania \\ 2 Department of Infectious Diseases, University of Medicine, Pharmacy, Science and Technology of Târgu Mureș, \\ Romania \\ 3 Department of Anesthesiology \& Pain, Medicine University of Washington Medical Center, Seattle WA, USA
}

\begin{abstract}
In recent years, a new form of medicine has become increasingly significant, namely, personalised medicine (PM). PM is a form of care in which treatment is tailored for an individual patient.

PM is about using multiple data sets to create a digital human mapping. A person's biological traits are determined by the interactions of hundreds of genes and gene networks, as well as external factors such as diet and exercise. Combining and then investigating these multiple databases with powerful statistical tools, allows a new understanding of how genetic intricacy drives health and disease and so leads to a closer personalised medical approach that targets each individual's unique genetic make-up.

Sepsis is a systemic inflammatory response to infection, ranging from systemic inflammatory response syndrome (SIRS) to septic shock and multiple organ dysfunction syndromes (MODS). Sepsis is the most common cause of death in intensive care patients. Treatments in an ICU may need to be adapted to the continuous and rapid changes of the disease, making it challenging to identify a single target. PM is thus seen as the future of sepsis treatment in the ICU. The fact that individual patients respond differently to treatment should be regarded as a starting point in the approach to providing treatment. The disease itself comes secondary to this concept.
\end{abstract}

Keywords: precision medicine, intensive care, sepsis, multiple datasets, genome

Received: 14 April 2019 / Accepted: 29 July 2019

\section{INTRODUCTION}

Hippocrates, the "Father" of modern medicine, was convinced that disease was a result of multiple factors, including the patient's lifestyle, environmental forces and diet. Accordingly, as these are unique for every individual, treatment should be focused on the individual. He stated that because the organism acts as one, it should be treated as one, and not as individual parts of a bigger system.

In recent times, a new form of medicine has become increasingly exercised, namely personalised medicine $(\mathrm{PM})$ and is efficacious in many medical specialities.

Several biomarkers with the potential for diagnosis and prognosis of different field-specific conditions, such as hypertension, atherosclerosis, and coronary bypass surgery, have already been identified as useful in cardiovascular PM medicine [1,2]. Oncology is anoth- er field in which PM is already widely implemented. A wide array of biomarkers are specific for different types of neoplasms and their evolution, allowing for the treatment of different forms of neoplasm to be tailored to the individual. Biomarkers, specific for melanomas [3], breast cancer [4], renal cancer [5], gastrointestinal disorders [6] orthopaedics [7], and postoperative care [8] have been employed these areas.

Pharmacogenomic biomarkers are currently accepted as key components in drug labelling.

Table 1. list the drugs and also the biomarkers which are related to those drugs.

They include germline or somatic gene variants, functional deficiencies with a genetic aetiology, gene expression differences, and chromosomal abnormalities; selected protein biomarkers that are used to select treatments for patients are also included [9]. 
Table 1. Table of Pharmacogenomic Biomarkers in Drug Labeling (10)

\begin{tabular}{|c|c|c|}
\hline Medical area & Drug & Biomarker \\
\hline Infectious & Abacavir & HLA-B \\
\hline \multirow[t]{4}{*}{ Diseases } & Boceprevir & IFNL3 (IL28B)- genetic variant near the gene encoding interferon-lambda-3 \\
\hline & Ceftriaxone & G6PD \\
\hline & Daclatasvir & IFNL3 (IL28B) \\
\hline & Efavirenz & CYP2B6 \\
\hline \multirow[t]{21}{*}{ Oncology } & Abemaciclib (1) & ESR (HR)-hormone receptor \\
\hline & Abemaciclib (2) & $\begin{array}{l}\text { ERBB2 (HER2)- , human epidermal growth factor receptor } 2 \\
\text { ERBB2 (HER2) }\end{array}$ \\
\hline & Ado-Trastuzumab Emtansine & EGFR (epidermal growth factor receptor) \\
\hline & Afatinib & ALK (anaplastic lymphoma kinase) \\
\hline & Alectinib & $\mathrm{CD} 274(\mathrm{PD}-\mathrm{L} 1)$ \\
\hline & Atezolizumab (1) & Gene Signature (T-effector) \\
\hline & Atezolizumab (2) & ROS \\
\hline & Crizotinib & BRAF \\
\hline & Dabrafenib & RAS \\
\hline & Dabrafenib & ESR, PGR (Hormone Receptor) \\
\hline & Docetaxel & CD274 (PD-L1) \\
\hline & Durvalumab & Chromosome $17 p$ \\
\hline & Duvelisib & FLT3 \\
\hline & Gilteritinib & Chromosome 11q \\
\hline & Ibrutinib & BCR-ABL1 (Philadelphia chromosome) \\
\hline & Inotuzumab Ozogamicin & Microsatellite Instability Mismatch Repair \\
\hline & Ipilimumab & IDH1- isocitrate dehydrogenase-1 \\
\hline & Ivosidenib & HLA-DQA1, HLA-DRB1 \\
\hline & Lapatinib & NTRK-gene \\
\hline & Larotrectinib & NUDT15- nucleotide diphosphatase \\
\hline & Mercaptopurine & UGT1A1-polymorphysm \\
\hline \multirow{4}{*}{ Neurology } & Amifampridine & NAT2 (N-acetyltransferase 2) \\
\hline & Citalopram & CYP2C19 \\
\hline & Inotersen & TTR \\
\hline & Nusinersen & Nusinersen \\
\hline \multirow[t]{2}{*}{ Psychiatry } & Amphetamine & CYP2D6 \\
\hline & Clozapine & CYP2D6 \\
\hline \multirow[t]{6}{*}{ Anesthesiology } & Articaine and Epinephrine (1) & G6PD \\
\hline & Chloroprocaine & G6PD \\
\hline & Codeine & CYP2D \\
\hline & Lidocaine and Tetracaine & G6PD \\
\hline & Lofexidine & CYP2D6 \\
\hline & Mivacurium & $\mathrm{BCHE}$ \\
\hline \multirow[t]{8}{*}{ Hematology } & Avatrombopag (1) & F2 (Prothrombin) \\
\hline & Avatrombopag (2) & F5 (Factor V Leiden) \\
\hline & Avatrombopag (3) & SERPINC1 (Antithrombin III) \\
\hline & Blinatumomab & BCR-ABL1 (Philadelphia chromosome) \\
\hline & Emapalumab-Izsg & $\begin{array}{l}\text { PRF1, RAB27A, SH2D1A, STXBP2, STX11, UNC13D, XIAP (Hemophagocy tic Lym- } \\
\text { phohistiocytosis) }\end{array}$ \\
\hline & Enasidenib & $\mathrm{IDH} 2$ \\
\hline & Lenalidomide & Chromosome $5 q$ \\
\hline & Lusutrombopag & PROC, SERPINC1 (Antithrombin III) \\
\hline \multirow{3}{*}{$\begin{array}{l}\text { Inborn Errors of } \\
\text { Metabolism }\end{array}$} & Cerliponase Alfa & TPP1- tripeptidyl peptidase 1 \\
\hline & Eliglustat & CYP2D6 \\
\hline & Migalastat & GLA-galactosidase alpha gene \\
\hline \multirow[t]{2}{*}{ Urology } & Fesoterodine & CYP2D6 \\
\hline & Tolterodine & CYP2D6 \\
\hline \multirow[t]{3}{*}{ Pulmonary } & Formoterol & CYP2D6 \\
\hline & & CYP2C19 \\
\hline & Ivacaftor & CFTR- cystic fibrosis transmembrane conductance regulator \\
\hline Gynecology & Ospemifene & CYP2C9 \\
\hline
\end{tabular}


$\mathrm{PM}$ is also well established in the field of genetics, where a vast array of biomarkers can be employed to identify specific modifications and facilitate in choosing a suitable treatment approach [10]. Current emphasis focuses on advances in genetic testing and biomarker identification. The possibility of creating a patient's "omic" (vide infra) appraisal and then using this data and information, establish a remarkable and unprecedented opportunity to develop individualised treatment strategies [11].

PM is an integration of complete biological systems which aims to create a stratified medicine with personalised care. Most medical doctors are working at the phenome level when treating a specific disease, be it cancer, cardiovascular conditions or psychiatric disorders. Occasionally treatment is extended to the genome level, which can identify rare variations of DNA or other genomic rearrangements. There are other "omes" zones including epigenome, transcriptome, proteome, metabolome though these are less commonly used. These zones contain tremendous information which could be utilised in treating the individual instead of the disease [10].

Personalised medicine is about using data on multiple scales, and having the ability to create a digital human mapping, with superimposed layers which includes, among other things, social graphs, biosensors, imaging for anatomy description, the characteristics of various "omics" such as genomics, DNA sequencing, transcriptomics, proteomics, metabolomics, and epigenomics. All these layers are wrapped in the nongenetic totality of human environment, the exposome [11]. All these elements allow the creation of a unique genetic print for every patient, individualised, a nonrepetitive print on which future medical decisions could be based.

\section{- PeRsonalised Medicine in ICU}

It is customary in today's approach to treatment, to create both standardise diagnosis and therapies. There are published guidelines and protocols, which are undeniably essential, but which in many instances do no more than to justify current therapeutic mindsets and approaches. At times, even existing legislative frameworks cannot solve every patient's problems. A change is needed to modify the baseline approach toward that of personalised, preventive, predictive, pharmacotherapeutics and patient participatory medicine.
Recent studies have classified ICU syndromes into subtypes. One way of creating these subtypes was by gene expression analysis, which helped differentiate sepsis from non-septic states [12] and also in estimating the likeliness of those subtypes to respond to specific treatments [13].

Specific genes were sequenced to identify single nucleotide polymorphisms associated with different outcomes in both sepsis and ARDS $[14,15]$. In genomewide association studies (GWAS) of sepsis and ARDS, consideration was given to adding precision and other pharmcogenes to the list of drugs used in sepsis [16]. Beyond genomics, precision medical information has been subtracted from other data types. Serum biomarkers have shown to be useful in differentiating septic patients with or without ARDS as well as classifying ARDS. [17] The recent identification of critical illness subtypes points to an emerging need for relating them to precise therapies. However, despite new advances in this new area of medicine, precision therapies in the ICU are at present, neither clearly defined nor generally accepted.

\section{DATA fOR PERSONAlised MEdicine}

Data concerning medical history and various prescribed treatments for each patient should be contained in an electronic patient health record (EHR). The ability to process such information is impeded by the sheer size of these data and requires the involvement of medical specialists working in conjunction with the latest computer technology. In order to achieve this goal, computer models are required to help clinicians organise the data, recognise patterns, and interpret results allowing the creation of specific models which could then be used for tailoring distinctive treatments for individual patients.

This "big data" in medicine is expected to have a significant role in pharmacogenetics and stratified healthcare. Nowadays, patients are treated in large cohorts based on their common diagnosis. However, each individual is different and can and will react differently to the specific treatment of a distinct disease [18-20].

There is ongoing research in this respect, one example being the Pan-Cancer project, initiated by the Cancer Genome Atlas research network which aims to analyse a multitude of tumour types and molecular modifications in cancer types, and to provide a starting point for newer discoveries in the field [18]. Other 
examples are the Cancer Cell Line Encyclopedia and the Genomics of Drug Sensitivity in Cancer projects which provide vast genomic databases for cancer cell abnormalities and their response to specific treatments $[19,20]$.

The MIMIC III database, a registry used to extract and process data for retrospective analysis, is a useful data registry created for use in ICU [21].

However, having raw data sets in not enough. These data need validation, storage, protection and specific processing in order to be useful for the creation of individualised treatments. To meet these requirements, suitable infrastructures and specialists are required [22].

The prerequisite to creating an EHR is that all data relating to a patient be combined and gathered in a single virtual file which can be accessed when a piece of medical information is needed. In creating an EHR, a joint effort is needed on the part of the patient, the medical specialists and different branches of research and informatics. (Figure 1)

There are sensitive issues in developing the bio-informatics, not the least involving the protection of data. Bioinformatics requires that a patient's specific medical data are known in order to have all the "necessary in- gredients" for preparing a specific if not unique treatment plan. This requirement may contravene existing data protection policies. For succeeding in creating a patient data bank, an interdisciplinary team is needed. As stated above, such a team should comprise, in addition to clinicians and IT specialists, ethicists and patient representatives.

\section{- Personalised Medicine in Sepsis}

Sepsis is a systemic inflammatory response to infection, ranging from systemic inflammatory response syndrome (SIRS) to septic shock and multiple organ dysfunction syndromes (MODS). Sepsis is the single most encountered cause of death in intensive care patients [23].

Different stages of sepsis lead to different dysfunctions, which are present systemically. SIRS determines endothelial dysfunction, impairment of microcirculation, hypoxia, apoptosis, multiple organ failure and death [24]. Sepsis, as a syndrome, is comprised of a cascade of pro and anti-inflammatory cytokines and mediators in the systemic circulation which are found at different stages of sepsis. Examples of the most common inflammatory cytokines in sepsis are tumour

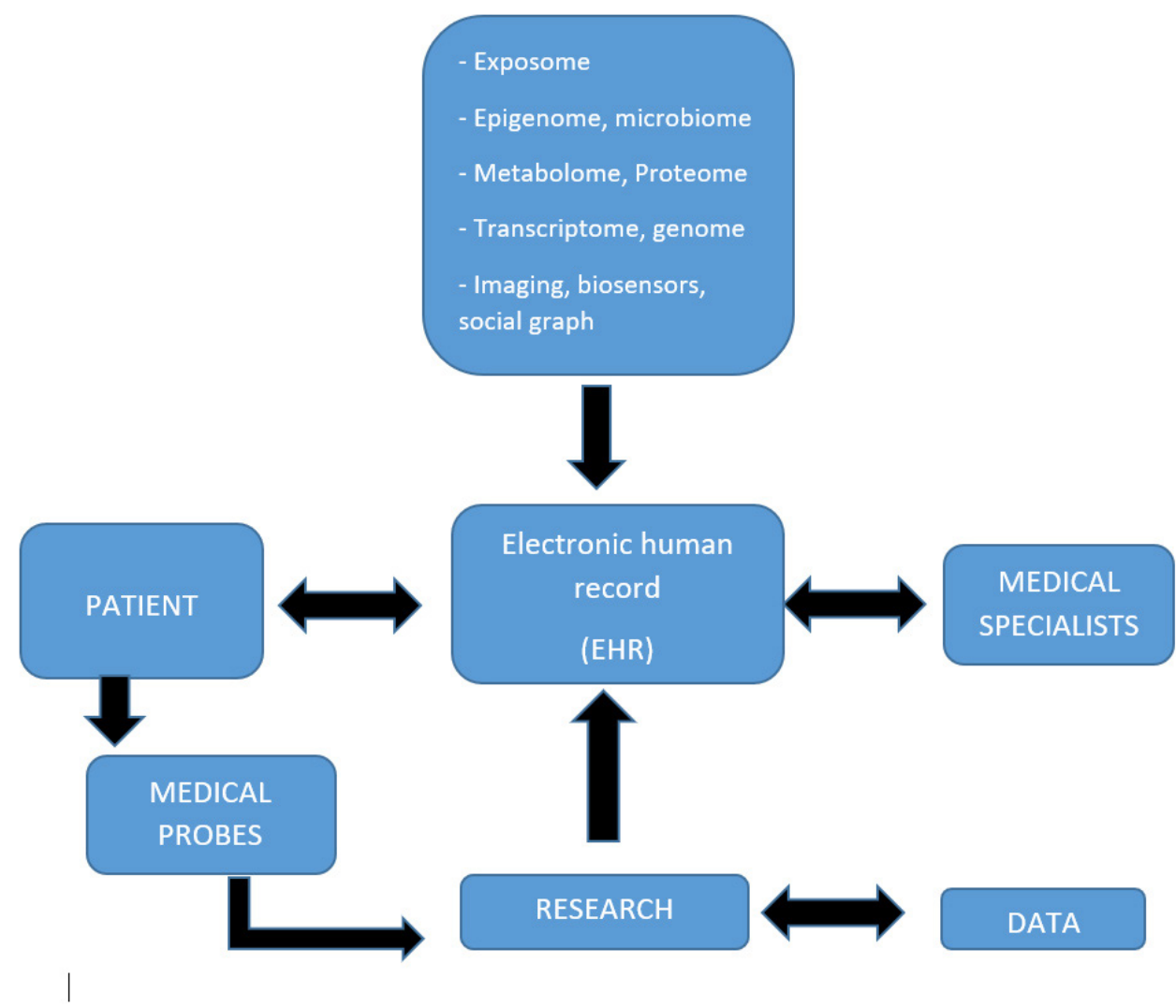

Fig. 1. Big data circuit 
necrosis factor- $\alpha$ (TNF- $\alpha$ ), interleukin-1 (IL-1) and pro-inflammatory cytokines IL-6 and IL-8. They are released by activating macrophages and CD4 $\mathrm{T}$ cell within the first hour after infection. To counteract and also to prevent severe damage, anti-inflammatory mediators such as IL-10, IL-13, IL-14, and transforming growth factor- $\beta$ (TGF- $\beta$ ), are also released [25].

Immunomodulatory therapies in which patients are prescribed corticosteroids, anti-TNF-aantibodies, anti-interleukin antibodies, platelet-activating factor (PAF) antagonists, antioxidants, as well as the use of selenium modulation of coagulation and complement pathway, have been accepted as beneficial [26-32], and indeed, some of these strategies proved to be efficacious upon septic patients survival of [33].

The clinical evolution characteristics of sepsis make both diagnosis and treatment difficult because patients proceed through different stages of sepsis, with each stage requiring treatment specifically targeted to counteract these variations. Sepsis patients are still treated as groups not as individuals, even though there are reported examples of treatment which are proven to be efficacious for septic patients but not others [34].

Seymour et al. (2016) outlined revised sepsis definitions based on large data sets obtained from several ICUs. This is seen as an initial step forward towards PM in the management of sepsis [34].

Prompt diagnosis and treatment of sepsis are crucial to reducing mortality. The mortality risk increases with every hour of delay in diagnosis and commencement of appropriate therapy. Prognostic scores are now used to assess mortality risk. The widely accepted Sequential Organ Failure Assessment (SOFA) score, assesses the severity of organ dysfunction in patients assumed to be septic [34]. However, because of the complexity of the method and the need of a large amount of medical data relating to the patient, which are not always available a new prognosis score, "quick SOFA" has been proposed. This scoring system is simplified, containing only three variables, and is targeted to ease the identification of septic patients at risk of death [34].

The main shortcoming in sepsis diagnosis is that there is no "gold standard" blood test, which can identify patients with infections before results become available from standard microbiological cultures.

The ongoing search for new therapies for sepsis and new prognostic and diagnostic biomarkers has generated several dozen microarray-based genome-wide expression studies over the past decade, variously focusing on the diagnosis, prognosis, pathogen response, and underlying sepsis pathophysiology. Despite tremendous gains in the understanding of gene expression in sepsis, few insights have translated to improvements in clinical practice $[35,36]$.

Most of the studies which were conducted on sepsis treatment had not ended with significant results regarding the survival of septic patients when the enrolment was based on heterogeneous groups of patients $[37,38]$.

\section{- CONCLUSION}

Precision medicine seeks to recognise the patient as an individual, as unique, using modern technology to store, access and analyse the vast amount of medical data.

Precision medicine is the future treatment approach in ICU, especially in sepsis treatment. The fact that patients respond differently to treatment procedures should be regarded as a starting point in the treatment of an individual.

\section{ACKNOWLEDGEMENT}

The research has been carried out within the project: The clinical relevance of coding genes polymorphisms cytokines (IL-6, IL-10, TNFalfa) and cellular receptors (CD14, TREM-1, TLR4) and PAI in sepsis, no. $17801 / 2 / 2015$, funded through internal research grants by the University of Medicine and Pharmacy of Tîrgu Mures, Romania.

\section{- CONFLICT OF INTEREST}

None to declare

\section{REFERENCES}

1. Vea A, Llorente-Cortes V, de Gonzalo-Calvo D. Circular RNAs: a novel tool in cardiovascular biomarker development? Noncoding RNA Investig. 2018; 2:39.

2. Kertai MD, Li YJ, Li YW et al. Genome-wide association study of perioperative myocardial infarction after coronary artery bypass surgery. BMJ Open. 2015; 6;5(5):e006920 doi: 10.1136/bmjopen-2014-006920

3. Valpione S, Martinoli C, Fava P, Mocellin S, Campana LG, Quaglino P. Personalized medicine: Development and external validation of a prognostic model for metastatic melanoma 
Available online at: www.jccm.ro

patients treated with ipilimumab. EJC. 2015; (14): 2086-94.

4. Molinaro, S, Pieroni S, Mariani F, Liebman M. Personalized medicine: Moving from correlation to causality in breast cancer. European Journal of Molecular \& Clinical Medicine. 2015; 2(2): 59

5. Stafford-Smith M, Li YJ, Mathew JP et al. Genome-wide association study of acute kidney injury after coronary bypass graft surgery identifies susceptibility loci. Kidney Int. 2015; 88(4):823-32.

6. Kunin A, PolivkaJr P, Moiseeva N, Golubnitschaja O. Dry mouth and "Flammer" syndromes-neglected risks in adolescents and new concepts by predictive, preventive and personalised approach. EPMA Journal.2018; 9:307-12.

7. Li X, Seebacher NA, Hornicek FJ, Xiao T, Duan Z. Application of liquid biopsy in bone and soft tissue sarcomas: Present and future. Cancer Lett. 2018; 439:66-77.

8. Maslove MD, Lamontagn L, Marshall JC, Heyland KD. A path to precision in the ICU. Critical Care.2017; 21:79-85.

9. Food and Drug Administration. Science and Research Drugs,Table of Pharmacogenomicbiomarkers.Available at:https://www.fda.gov/downloads/Drugs/ScienceResearch/ UCM578588.pdf

10. National Research Council. Toward Precision Medicine: Building a Knowledge Network for Biomedical Research and a New Taxonomy of Disease. Available at https://www.nap. edu/catalog/13284/toward-precision-medicine-building-aknowledge-network-for-biomedical-research

11. Topol EJ. Individualized medicine from prewomb to tomb. Cell. 2014; 157(1):241-53.

12. Sweeney TE, Shidham A, Wong HR, Khatri P. A comprehensive time-course-based multi cohort analysis of sepsis and sterile inflammation reveals a robust diagnostic gene set. Sci Transl Med. 2015;7(287):287ra71 doi:10.1126/scitranslmed. aaa5993

13. Wong HR, Atkinson SJ, Cvijanovich NZ, et al. Combining Prognostic and Predictive Enrichment Strategies to Identify Children With Septic Shock Responsive to Corticosteroids. Crit Care Med. 2016;44(10):e1000-e1003. doi:10.1097/ CCM.0000000000001833.

14. Walley KR, Thain KR, Russell JA, et al. PCSK9 is a critical regulator of the innate immune response and septic shock outcome. Sci Transl Med. 2014;6(258):258ra143. doi:10.1126/ scitranslmed.3008782

15. Sapru A, Liu KD, Wiemels J et al. Association of common genetic variation in the protein $\mathrm{C}$ pathway geneswith clinical outcomes in acute respiratory distress syndrome. Crit Care.2016; 20(1):151

16. Russell JA. Genomics and pharmacogenomics of sepsis: so close and yet so far. Crit Care. 2016;1-4.

17. Calfee CS, Janz DR, Bernard GR et al. Distinct molecular phenotypes of direct vs indirect ARDS in single-center and multicenter studies. Chest. 2015; 147:1539-48.

18. Weinstein JN, C Collisson EA, Mills GB, Shaw KR, Ozenberger
The Journal of Critical Care Medicine 2019;5(3) • 95

BA, Ellrott K. The cancer Genome Atlas Pan-Cancer analysis project. Nat Genet.2013; 45(10): 1113-20.

19. Barretina, Caponigro G, Stransky N et al. The Cancer Cell Line Encyclopedia enables predictive modelling of anti-cancer drug sensitivity. Nature. 2012; 483(7391): 603-7.

20. Garnett MJ, Sys Edelman EJ, Heidorn SJ et al. Systematic identification of genomic markers of drug sensitivity in cancer cells. Nature. 2012; 483(7391): 570-5.

21. Johnson AEW, Pollard TJ, Shen L, et al. MIMIC-III, a freely accessible critical care database. Sci Data.2016; 3:160035. doi: 10.1038/sdata.2016.35

22. Shankar-Hari M, Phillips GS, Levy ML et al. Developing a new definition and assessing new clinical criteria for septic shock. JAMA. 2016; 315(8):775-87.

23. Mayr FB, Yende S, Angus DC. Epidemiology of severe sepsis. Virulence 2014; 5:4-11.

24. Alberti C, Brun-Buisson C, Chevret S et al. Systemic inflammatory response and progress into severe sepsis in critically ill infected patients. Am J Resp Crit Care Med. 2005; 171:461-8.

25. King EG, Bauzá GJ, Mella JR, Remick DG. Pathophysiologic mechanisms in septic shock. Lab Invest 2014; 94:4-12.

26. Katsenos CS, Antonopoulou AN, Apostolidou EN et al. Early administration of hydrocortisone replacement after the advent of septic shock: impact on survival and immune response. Crit Care Med.2014; 42(7):1651-7.

27. Lv S, Han M, Yi R, Kwon S, Dai C, Wang R. Anti-TNF- $\alpha$ therapy for patients with sepsis: a systematic meta-analysis. Int J ClinPract. 2014; 68(4):520-8.

28. Shindo $Y$, Unsinger J, Burnham C-A, Green JM, Hotchkiss RS. Interleukin-7 and anti-programmed cell death 1 antibody have differing effects to reverse sepsis-induced immunosuppression. Shock. 2015; 43(4):334-43.

29. Kowalska MA, Zhao G, Zhai L et al. Modulation of protein C activation by histones, platelet factor 4 , and heparinoids new insights into activated protein $\mathrm{C}$ formation. Arterioscler Thromb Vasc Biol.2014; 34:120-6.

30. Schomburg L. Selenium in sepsis-substitution, supplementation or pro-oxidative bolus? Crit Care. 2014; 18:444. doi: 10.1186/ cc13963

31. Wilson JX. Antioxidants in sepsis. In: Systems biology of free radicals and antioxidants. Springer. 2014: 3267-72.

32. Lupu F, Keshari RS, Lambris JD, Coggeshall KM. Crosstalk between the coagulation and complement systems in sepsis. Thromb Res. 2014; 133(1):S28-S31.

33. Hutchins NA, Unsinger J, Hotchkiss RS, Ayala A. The new normal: immunomodulatory agents against sepsis immune suppression. Trends Mol Med. 2014; 20(4):224-33.

34. Seymour CW, Liu VX, Iwashyna TJ et al. Assessment of Clinical Criteria for Sepsis: For the Third International Consensus Definitions for Sepsis and Septic Shock (Sepsis-3). JAMA. 2016; 315(8):762-74. 
96 The Journal of Critical Care Medicine 2019;5(3)

35. Wong HR, Wheeler DS, Tegtmeyer K, et al. Toward a clinically feasible gene expression-based subclassification strategy for septic shock: proof of concept. Crit Care Med. 2010;38(10):1955-61.

36. Joehanes $\mathrm{R}$, Zhang $\mathrm{X}$, Huan $\mathrm{T}$, et al. Integrated genome-wide analysis of expression quantitative trait loci aids interpretation of genomic association studies. Genome Biol. 2017;18(1):16.
Available online at: www.jccm.ro

37. Seymour CW, Gesten F, Prescott HC, et al. Time to Treatment and Mortality during Mandated Emergency Care for Sepsis. N Engl J Med. 2017;376(23):2235-44.

38. László I, Trásy D, Molnár Z, Fazakas J. Sepsis: From Pathophysiology to Individualized Patient Care. J Immunol Res. 2015;2015:510436. 